\title{
The solar system: a laboratory for the study of the physics of particle acceleration
}

\author{
Robert P. Lin \\ Physics Department and Space Sciences Laboratory, \\ University of California, Berkeley CA 94720-7450, USA \\ email: rlin@ssl.berkeley.edu
}

\begin{abstract}
A remarkable variety of particle acceleration occurs in the solar system, from lightningrelated acceleration of electrons to tens of $\mathrm{MeV}$ energy in less than a millisecond in planetary atmospheres; to acceleration of auroral and radiation belt particles in planetary magnetospheres; to acceleration at planetary bow shocks, co-rotating interplanetary region shocks, shocks driven by fast coronal mass ejections, and possibly at the heliospheric termination shock; to acceleration in magnetic reconnection regions in solar flares and at planetary magnetopause and magnetotail current sheets. These acceleration processes often occur in conjunction with transient energy releases, and some are very efficient. Unlike acceleration processes outside the solar system, the accelerated particles and the physical conditions in the acceleration region can be studied through direct in situ measurements, and/or through detailed imaging and spectroscopy. Here I review recent observations of tens of $\mathrm{MeV}$ electron acceleration in the Earth's atmosphere and in the Earth's radiation belts, electron and ion acceleration related to magnetic reconnection in solar flares, electron acceleration to $\geqslant 300 \mathrm{keV}$ in magnetic reconnection regions in the Earth's deep magnetotail, and acceleration of solar energetic particles (SEPs) by shocks driven by fast coronal mass ejections (CMEs).
\end{abstract}

Keywords. acceleration of particles in solar system

\section{Terrestrial gamma-ray flashes (TGFs)}

Bursts of $\sim 30$ to $>300 \mathrm{keV} \gamma$-rays from the Earth lasting $\sim 1 \mathrm{~ms}$, were first detected by the BATSE instrument (designed to detect cosmic $\gamma$-ray bursts, CGRBs) on the Compton Gamma-Ray Observatory (Fishman et al. 1994). 75 TGFs were detected over nine years, and it was shown that they occurred simultaneous with lightning. Recent observations from the RHESSI mission showed that TGFs typically extend up to > $10 \mathrm{MeV}$ in energy; the spectrum obtained by averaging over many bursts is consistent with bremsstrahlung of a minimum of $\sim 10^{15}$ electrons of $\sim 30 \mathrm{MeV}$ energy (Smith et al. 2005). The RHESSI observations show no energy dispersion down to $\sim 0.1 \mathrm{~ms}$ time scale, indicating that the acceleration is extremely fast. About 10-15 TGFs are detected a month; the much higher rate is likely due to the fact that RHESSI records every photon while BATSE depends on a trigger algorithm that is optimized for CGRBs. Since RHESSI can only detect TGFs within line of sight, this implies an order of a thousand TGFs per day over the latitude band from -38 to +38 degrees. The TGFs are definitely associated with lightning, and appear to be preferentially observed in the tropics. The present hypothesis for TGFs is that they are due to runaway of cosmic ray secondary relativistic electrons in the very large potential ( $\sim 30$ megavolts) above the cloud set up by the lightning stroke. Electrons at energies of a few MeV have the lowest energy loss to Coulomb collisions and therefore require the smallest electric field to run away, but many questions remain. It is remarkable that the Earth can be a high energy accelerator and gamma-ray source, and 
it should be noted that lightning orders of magnitude more intense has been detected (through the associated radio emission) from Jupiter and Saturn.

\section{Radiation belt $\mathrm{MeV}$ electron acceleration}

In recent years the importance of transients in the acceleration of radiation belt particles has become more apparent. Spectrogram plots of the MeV electron fluxes vs. equatorial distance as a function of time are dominated by sudden ( $\leqslant 1$ day) increases followed by decay over months, with more intense fluxes around solar minimum when high speed streams are present. Thus, the transient response to high-speed solar wind streams appears to accelerate much of the radiation belt electrons through a combination of injection of particles from the magnetotail followed by inward radial diffusion driven by ULF waves. Much rarer are situations where a strong shock from a large CME impinging on the magnetosphere produces an intense transient electric field which is able to accelerate electrons up to $>10-15 \mathrm{MeV}$ in an electron drift period (such as occurred on March 24, 1991). These events, however, produce the highest energy electron belts, and those can last for years.

\section{Solar flare electron and ion acceleration associated with magnetic reconnection}

It has become clear from RHESSI imaging spectroscopy measurements of solar flares that the energy release process for solar flares, e.g., magnetic reconnection, is able to efficiently accelerate ions and electrons to high energies. The closely similar temporal variations of pairs of hard X-ray (HXR) footpoints seen in large $\gamma$-ray flares such as the 23 July 2002 provide strong evidence for reconnection and the formation of new closed loops (Lin et al. 2003). The temporal variation of the HXR intensity also shows a rough correlation with the rate of reconnection, as inferred from the footpoint motions, suggesting that the energy released goes into the acceleration of the HXR-producing electrons. For the first time, the accelerated ions were located through RHESSI imaging of the $2.223 \mathrm{MeV}$ neutron-capture $\gamma$-ray line. Surprisingly, the line emission comes from a different location, $\sim 15,000 \mathrm{~km}$ away from the HXR emission, indicating that acceleration and/or propagation of the electrons and ions are different. In the large flare of 28 October 2003 two footpoint sources were observed in the gamma-ray line image, but they were again separated from the HXR footpoint sources by $\sim 10,000 \mathrm{~km}$ (Hurford et al. 2006). How electrons and ions are efficiently accelerated to high energies in the reconnection process is presently unknown.

\section{Electron acceleration in the magnetotail reconnection region}

The Wind spacecraft has provided in situ measurements in traversing the ion diffusion region (where the ions decouple from the magnetic field because of their large gyroradii) of a magnetic reconnection event observed deep ( $\sim 60$ Earth radii) in the Earth's magnetotail. Electrons up to $\geqslant 300 \mathrm{keV}$ were detected peaking in the ion diffusion region, with fluxes decreasing and the spectrum softening with distance away (Oieroset et al. 2002). No acceleration of ions was detected, however, and no unusual plasma wave activity was detected. Again the acceleration mechanism is presently unknown. 


\section{Solar energetic particle (SEP) acceleration by shocks}

SEPs are hypothesized to be accelerated by shocks driven by fast $(\sim 1-3$ thousand $\mathrm{km} / \mathrm{s}$ ) Coronal Mass Ejections (CMEs). In large SEP events, the efficiency, defined as the percentage of the total energy in the CME (dominated by the kinetic energy of the CME) that is in SEPs, is typically of order $\sim 10 \%$ (Emslie et al. 2004). This efficiency is also what is needed for supernova shocks to accelerate galactic cosmic rays. Fast CMEs and shocks are observed at $1 \mathrm{AU}$ on occasion, sometimes with coincident increases in SEP ions up to $\sim 10 \mathrm{MeV}$. Above $\sim 20 \mathrm{MeV}$, however, SEP increases related to interplanetary shocks near $1 \mathrm{AU}$ are almost never observed, implying that acceleration to those energies does not occur for some reason. The time profiles for SEP fluxes at high energies indicate that those particles are accelerated close to the Sun. Some very fast $(>2000 \mathrm{~km} / \mathrm{s})$ and wide $(\geqslant 100$ degrees) CMEs are unaccompanied by SEP events, and for a given CME speed, the peak SEP fluxes can vary by several orders of magnitude. Clearly, our understanding of the shock acceleration process is, at best, qualitative. NASA's planned Solar Sentinels mission is intended specifically to make progress on this fundamental problem of shock acceleration to high energies, with in situ measurements of pristine, freshly accelerated SEPs and of the unevolved shock and upstream waves that are presumably key to the acceleration process, and close $(0.25 \mathrm{AU})$ to the near-Sun acceleration region to avoid the blurring effects of scattering and diffusion of the SEPs in reaching $1 \mathrm{AU}$.

\section{References}

Emslie, A.G., Kucharek, H., Dennis, B.R., et al. 2004, J. Geophys. Res., 109, A10104

Fishman, G.J., Bhat, P.N., Mallozzi, R., et al. 1994, Science, 264, 1313

Hurford, G., Krucker, S., Lin, R.P., et al. 2006, ApJ (Letters), 644, L93

Lin, R.P., Krucker, S., Hurford, G.J., et al. 2003, ApJ (Letters), 595, L69

Oieroset, M., Lin, R.P., Phan, T.D., et al. 2002, Phys. Rev. Lett., 89, 195001

Smith, D.M., Lopez, L.I., Lin, R.P., \& Barrington-Leigh, C.P. 2005, Science, 307, 1085 